\title{
Hospital infection and COVID-19: Do not put all your eggs on the "swab" tests
}

\author{
Francesco Chirico MD ${ }^{1,2}$ (1), Gabriella Nucera $\mathrm{MD}^{3,4}$ and Nicola Magnavita $\mathrm{MD}^{5,6}$ \\ ${ }^{1}$ Postgraduate School of Occupational Medicine, Università Cattolica del Sacro Cuore, Rome, Italy, ${ }^{2}$ Health Service Department, State Police, Ministry of Interior, \\ Milan, Italy, ${ }^{3}$ Faculty of Nursing, University of Milan, Italy, ${ }^{4}$ Fatebenefratelli Sacco, FatebeneFratelli Hospital, Milan, Italy, ${ }^{5}$ Postgraduate School of Occupational \\ Medicine, Università Cattolica del Sacro Cuore, Rome, Italy and ' ${ }^{6}$ Department of Woman/Child and Public Health, Fondazione Policlinico "A.Gemelli" IRCCS, \\ Rome, Italy
}

To the Editor-In healthcare settings, including long-term care facilities, hospital administrators have a legal obligation to set up a risk assessment strategy to carry out effective prevention and control measures during the management of suspected and confirmed cases of COVID-19 infection. ${ }^{1}$ Hospitalized inpatients and residents in care homes are often elderly and immunedepressed patients with comorbidities; thus, they are at high risk of infection and mortality. Special attention and efforts to protect or reduce transmission should be also applied in healthcare providers because depletion of the healthcare workforce not only will affect health care but also will contribute to the spread of the outside hospitals.

According to European Centres for Disease Control and Prevention (EU-CDC) guidelines, ${ }^{1}$ each hospital should constitute a 'COVID-19 preparedness and response committee' and should prepare a specific plan, including a number of administrative and structural measures for patient and healthcare management. Undoubtedly, the most important measure in reding the likelihood of nosocomial infection is early isolation of patients with COVID19 , or at least maintaining a safe distance between those who are awaiting diagnosis. However, a number of recent studies showed that patients with mild or nonspecific symptoms can escape isolation and thus introduce SARS-CoV-2 into hospitals, leading to clusters of nosocomial infections. ${ }^{2}$

To minimize the risk of spreading, mass testing with nasopharyngeal and oropharyngeal (NP/OP) swab of all patients has been proposed, ${ }^{2,3}$ associated with mass testing of both symptomatic and asymptomatic healthcare workers. ${ }^{4}$ Even the use of these expensive and demanding mass strategies, however, cannot be considered a measure of absolute guarantee.

Indeed, Xie et $\mathrm{al}^{5}$ observed typical COVID-19 chest lesions via computed tomography (CT) scans in 5 patients with a negative or weakly positive swab test (RT-PCR test). Another patient with a chest X-ray showing interstitial pneumonia but with a negative RT-PCR test was reported by Winichakoon et al. ${ }^{6}$ Kumar et $\mathrm{al}^{7}$ reported the case of a patient with pneumonia and negative nasopharingeal swab who tested positive some days later with a bronchial lavage sample. Bandirali et $\mathrm{al}^{8}$ found that asymptomatic or minimally symptomatic patients may have abnormalities in chest $\mathrm{X}$-rays after 14 days of quarantine, with a sensitivity of RT-PCR testing of $59 \%{ }^{8}$

Author for correspondence: Prof Francesco Chirico, E-mail: medlavchirico@ gmail.com

Cite this article: Chirico F, Nucera G, and Magnavita N. (2021). Hospital infection and COVID-19: Do not put all your eggs on the "swab" tests. Infection Control \& Hospital Epidemiology, 42: 372-373, https://doi.org/10.1017/ice.2020.254
Cao et $\mathrm{al}^{9}$ observed that patients with negative to RT-PCR tests may have specific IgG and/or IgM for SARS-CoV-2 at recovery stage. $^{9}$ In reality, the sensitivity of an NP/OP swab in the course of disease ranges between $42 \%$ and $71 \%{ }^{6}$ and depends on sampling technique, timing within the clinical course of COVID-19, and viral loads detected in the swab. ${ }^{6}$

In conclusion, given the fact that negative NP/OP swabs do not rule out COVID-19 diagnosis, we propose that all the patients hospitalized with pneumonia be subjected to swab obtained by deep tracheal aspirate, which has a lower risk of aerosolization. ${ }^{10}$ We further recommend that suspected infection be checked with a combination of repeated RT-qPCR tests and chest CT scan. All patients hospitalized without respiratory symptoms should also be checked with repeated RT-qPCR tests and chest X ray before admission in hospital wards.

Moreover, healthcare providers should be tested regularly with serological test and swabs and symptom monitoring. Finally, a policy of universal masking and eye shielding for all healthcare providers involved in direct patient care is needed.

Acknowledgments. None.

Financial support. No financial support was provided relevant to this article.

Conflicts of interest. All authors report no conflicts of interest relevant to this article.

\section{References}

1. Infection prevention and control and preparedness for COVID-19 in healthcare settings - third update. EU-CDC European Centre For Disease Prevention and Control. https://www.ecdc.europa.eu/en/publications-data/ infection-prevention-and-control-and-preparedness-covid-19-healthcaresettings. Accessed May 22, 2020.

2. Klompas M. Coronavirus disease 2019 (COVID-19): protecting hospitals from the invisible. Ann Intern Med 2020 Mar 11 [Epub ahead of print]. doi:10.7326/M20-0751.

3. Gandhi M, Yokoe DS, Havlir DV.Asymptomatic transmission, the Achilles' heel of current strategies to control COVID-19. N Engl J Med 2020 Apr 24 [Epub ahead of print]. doi:10.1056/NEJMe2009758.

4. Black JRM, Bailey C, Przewrocka J, Dijkstra KK, Swanton C. COVID-19: the case for health-care worker screening to prevent hospital transmission [published correction appears in Lancet 2020 Apr 17]. Lancet 2020;395: 1418-1420.

5. Xie X, Zhong Z, Zhao W, Zheng C, Wang F, Liu J. Chest CT for typical 2019-nCoV pneumonia: relationship to negative RT-PCR testing. Radiology 2020 Feb 12 [Epub ahead of print]. doi:10.1148/radiol.2020 200343.

(c) 2020 by The Society for Healthcare Epidemiology of America. All rights reserved. This is an Open Access article, distributed under the terms of the Creative Commons Attribution licence (http://creativecommons.org/licenses/by/4.0/), which permits unrestricted re-use, distribution, and reproduction in any medium, provided the original work is properly cited. 
6. Winichakoon P, Chaiwarith R, Liwsrisakun C, et al. Negative nasopharyngeal and oropharyngeal swab does not rule out COVID-19. J Clin Microbiol 2020. doi: 10.1128/JCM.00297-20

7. Kumar DS, O'Neill SB, Johnston JC, Grant JM, Sweet DD. SARS-CoV-2 infection in a 76-year-old man with negative results for nasopharyngeal swabs and possible nosocomial transmission. CMAJ 2020 Apr 24 [Epub ahead of print]. doi:10.1503/cmaj.200641.

8. Bandirali M, Scofienza LM, Serra R, et al. Chest X-ray findings in asymptomatic and minimally symptomatic quarantined patients in Codogno,
Italy. Radiology 2020 Mar 27 [Epub ahead of print. doi:10.1148/radiol. 2020201102.

9. Cao G, Tang S, Yang D, et al. One nosocomial cluster following with a familial cluster of COVID-19 cases: the potential transmission risk in patients with negative swab tests. 2020 Mar [Preprint]. doi:10.21203/rs.3.rs-17879/v1.

10. Alhazzani W, Møller MH, Arabi YM, et al. Surviving sepsis campaign: guidelines on the management of critically ill adults with coronavirus disease 2019 (COVID-19). Intensive Care Med 2020 Mar 28 [Epub ahead of print]. doi: 10.1007/s00134-020-06022-5.

\title{
The value of hospital personnel serological screening in an integrated COVID-19 infection prevention and control strategy
}

\author{
Filippo Quattrone $M D^{1,2,3, a}$ (1), Marco Vabanesi $M D^{4, a}$ (1) , Alice Borghini $M D^{5}$, Giuseppe De Vito $M D^{6}$, \\ Michele Emdin PhD, MD ${ }^{1,3}$ and Claudio Passino MD ${ }^{1,3}$ \\ ${ }^{1}$ Institute of Life Sciences, Scuola Superiore Sant'Anna, Pisa, Italy, ${ }^{2}$ Department of Translational Research and New Technologies in Medicine and Surgery, \\ University of Pisa, Pisa, Italy, ${ }^{3}$ Fondazione Toscana Gabriele Monasterio, Pisa, Italy, ${ }^{4}$ Department of Neurology, San Raffaele Scientific Institute and University \\ Hospital, Milan, Italy, Institute of Management, Scuola Superiore Sant'Anna, Pisa, Italy and ${ }^{6}$ Fondazione Stella Maris, Pisa, Italy
}

To the Editor-Severe acute respiratory syndrome coronavirus 2 (SARS-CoV-2) is highly infectious in healthcare-related settings, both among patients and healthcare workers (HCWs). ${ }^{1}$ Hospital personnel have shown an increased risk of coronavirus disease 2019 (COVID-19) compared to the general population, possibly associated with repeated exposures and, in the current emergency context, frequent lack of adequate personal protective equipment (PPE). The spread of SARS-CoV-2 has also been dramatically efficient in long-term care facilities (LTCFs), where the combination of asymptomatic or paucisymptomatic occupational carriers and a highly fragile elderly population have produced numerous outbreaks, greatly contributing to the total burden of COVID-19related deaths. ${ }^{2}$

An integrated COVID-19 infection and prevention control (IPC) strategy must be promptly adopted by healthcare facilities to prevent further outbreaks. This strategy should involve HCWs, patients, visitors, and support personnel (eg, administrative and ancillary services workers) due to their reported role in nosocomial outbreaks. ${ }^{3}$ Current recommendations ${ }^{4}$ include the adoption of general IPC measures (eg, hand hygiene, physical distancing, universal use of surgical masks, and triage at entrance for fever, respiratory symptoms, and history of exposure to the virus), environmental measures (eg, enhanced surface cleaning, control of indoor air, proper linen, laundry, and waste management), administrative measures (eg, limiting visitor access and promoting remote work and telemedicine), and patient management measures (eg, dedicated pathways and isolation wards for patients with fever and respiratory symptoms, consistent use of adequate PPE, and universal SARS-CoV-2 screening for inpatients).

Author for correspondence: Filippo Quattrone, E-mail: filippo.quattrone@santannapisa.it a Authors of equal contribution.

Cite this article: Quattrone F, et al. (2021). The value of hospital personnel serological screening in an integrated COVID-19 infection prevention and control strategy. Infection Control \& Hospital Epidemiology, 42: 373-374, https://doi.org/10.1017/ice.2020.242
Systematic screening of HCWs and support personnel plays a key role in limiting the intrahospital spread of SARS-CoV-2. The most described approach is screening with viral genome real-time polymerase chain reaction (RT-PCR) on nasopharyngeal swabs. RT-PCR tests should be offered whenever an HCW presents with any symptom suggestive of COVID-19; initial screening is warranted for all new employees. Universal RT-PCR screening protocols $^{1,5}$ have shown promising results. A limitation of this approach is the short-term RT-PCR positivity, with consequent need of repeated testing, sustained usage of intensive laboratory resources, exposure risks for the operators involved in screening, and possibly reduced compliance with repeated swab testing. To overcome some defects of traditional PCR-based testing, novel kits for point-of-care rapid PCR testing are currently being developed, with as yet uncertain yield.

Antibody response to SARS-CoV-2 has not been completely characterized; however, from the best available data, ${ }^{6}$ it appears that the detection of serum anti-SARS-CoV-2 IgG antibodies with appropriate methods (ie, chemiluminescence enzyme immunoassay, CLIA) is observable in almost all infected subjects within 20 days from symptom onset. Data on IgM appear less conclusive, and currently, these data do not support the classic sequential IgM-IgG transition; therefore IgM should not be the only target of antibody search.

Many regulatory institutes have assessed rapid, point-of-care antibody tests based on lateral flow immunoassay (LFIA), produced by multiple manufacturers. Although tempting for practical reasons, these tests have not met expectations for use in clinical settings due to unsatisfactory sensitivity and specificity.

Serial serological screening with a validated technique, such as CLIA, could provide a significant contribution to IPC in hospitals and LTCFs, considering its lower cost, easier repeatability, and sustainability in the medium term, compared with swab-based molecular assays. Although serological tests have limited utility in diagnosing individual acute infections, they can inform actions to protect the hospital community. A serum antibody 